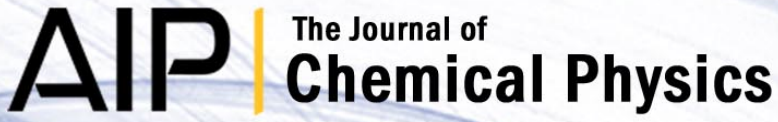

Influence of the repulsive Coulomb barrier on photoelectron spectra and angular distributions in a resonantly excited dianion

Daniel A. Horke, Adam S. Chatterley, and Jan R. R. Verlet

Citation: J. Chem. Phys. 139, 084302 (2013); doi: 10.1063/1.4818597

View online: http://dx.doi.org/10.1063/1.4818597

View Table of Contents: http://jcp.aip.org/resource/1/JCPSA6/v139/i8

Published by the AIP Publishing LLC.

Additional information on J. Chem. Phys.

Journal Homepage: http://jcp.aip.org/

Journal Information: http://jcp.aip.org/about/about_the_journal

Top downloads: http://jcp.aip.org/features/most_downloaded

Information for Authors: http://jcp.aip.org/authors

\section{ADVERTISEMENT}

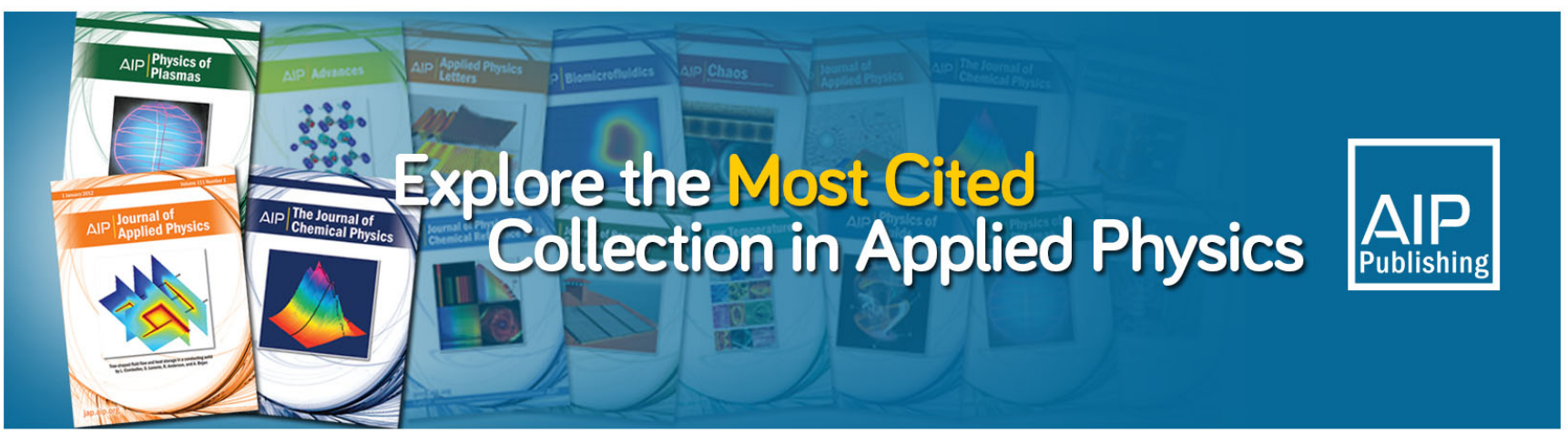




\title{
Influence of the repulsive Coulomb barrier on photoelectron spectra and angular distributions in a resonantly excited dianion
}

\author{
Daniel A. Horke, ${ }^{1, a)}$ Adam S. Chatterley, ${ }^{1,2}$ and Jan R. R. Verlet ${ }^{1, b)}$ \\ ${ }^{1}$ Department of Chemistry, University of Durham, Durham DH1 3LE, United Kingdom \\ ${ }^{2}$ Department of Chemistry, University of Warwick, Coventry CV4 7AL, United Kingdom
}

(Received 10 June 2013; accepted 2 August 2013; published online 22 August 2013)

\begin{abstract}
A photoelectron imaging study of the gas-phase dianion of pyrromethene-556 is presented. The photoelectron spectra and angular distributions following resonant excitation of the $S_{1}$ excited state with nanosecond and femtosecond laser pulses are compared, and the influence of the repulsive Coulomb barrier (RCB) in both cases evaluated. Photoelectron angular distributions show the effect of molecular alignment due to an allowed electronic excitation and can be understood qualitatively based on the calculated RCB surface using the Local Static Approximation. Comparison between femtosecond and nanosecond excitation reveals marked differences in the photoelectron spectra. While femtosecond experiments confirm that tunneling through the RCB is adiabatic, nanosecond experiments show a broad photoelectron feature peaking near the RCB maximum. This is explained in terms of the lifetime of internal conversion, which has been determined by time-resolved photoelectron spectroscopy to be $\sim 120$ ps: as this is faster than the nanosecond laser pulses, multiple photons can be absorbed through the $S_{1} \leftarrow S_{0}$ transition which leads to large amounts of internal energy and enables electron detachment directly above the RCB. Fragmentation and detachment from the monoanion are also inferred by the presence of photoelectrons emitted at very low kinetic energy. Our results highlight the difficulty in interpreting photoelectron spectra of polyanions in which a resonant state is excited. (C) 2013 AIP Publishing LLC. [http://dx.doi.org/10.1063/1.4818597]
\end{abstract}

\section{INTRODUCTION}

Gas-phase molecular polyanions (also commonly referred to as multiply charged anions or multianions) have received considerable scientific attention in recent years. ${ }^{1-3}$ This is partly due to the ubiquity of these systems in the condensed phase and in nature, but also due to their unusual electronic properties when isolated. While the presence of multiple repelling charges induces an inherent instability in an isolated polyanion, there is also an inherent electronic stability present. ${ }^{1,4-10}$ This arises from the balance of Coulomb repulsion between the excess negative charges and the attraction between the charges and the molecular nuclei. The result is the formation of a repulsive Coulomb barrier (RCB) to electron detachment. ${ }^{10-14}$ The presence of the RCB has led to the observation of phenomena such as polyanions with negative binding energy $y^{9,15}$ and low-energy cutoffs in photoelectron (PE) spectra, below which no electrons can be emitted directly. ${ }^{16}$

The exact shape and height of the RCB are strongly dependent on the location of excess charges within the molecular skeleton and can strongly affect the direction of detached photoelectrons. Photoelectron angular distributions (PADs) measured in PE imaging experiments can therefore be used

\footnotetext{
${ }^{a)}$ Present address: Center for Free-Electron Laser Science, DESY, Notkestr. 85, 22607 Hamburg, Germany.

b) Author to whom correspondence should be addressed. Electronic mail: j.r.r.verlet@durham.ac.uk
}

as a probe for the shape of the RCB, ${ }^{17-20}$ while the PE spectrum yields information about the RCB height. ${ }^{8}$

Time-resolved studies of the RCB have only recently been reported, in which electron tunneling through the barrier has been observed to occur on characteristic time-scales of a few to hundreds of picoseconds. ${ }^{5,21-23}$ Recently, our group has shown that this tunneling process is strongly adiabatic, conserving internal energy during the tunneling event. ${ }^{21}$ Hence, the electron tunneling can be viewed as a diagonal transition and consequently the usual correlation between the electron binding energy $(e B E)$ and electron kinetic energy $(e K E), e B E=h v-e K E$, is not necessarily valid. This was shown to be the case for the doubly deprotonated fluorescein dianion, ${ }^{21}$ but is probably quite general and has very recently also been observed in other systems. ${ }^{17,22,23}$ The current study aims to provide more detailed insight into this behavior and how the PE spectra and PADs produced following resonant tunneling are sensitive to the pulse duration of the excitation laser field.

PADs in polyanions are potentially very informative because the anisotropy of the RCB, which is determined by the location of the excess charges, can influence the trajectories of the outgoing PE. We have recently demonstrated that such information can be attained from an aligned sample created through resonant electronic excitation of a polyanion. ${ }^{17}$ The resultant PADs were shown to be dynamic, because the resonant alignment is lost as the initially created rotational wavepacket dephases with time. In this contribution we will also expand on these previously reported results, by considering in detail the shape of the RCB and we provide a 


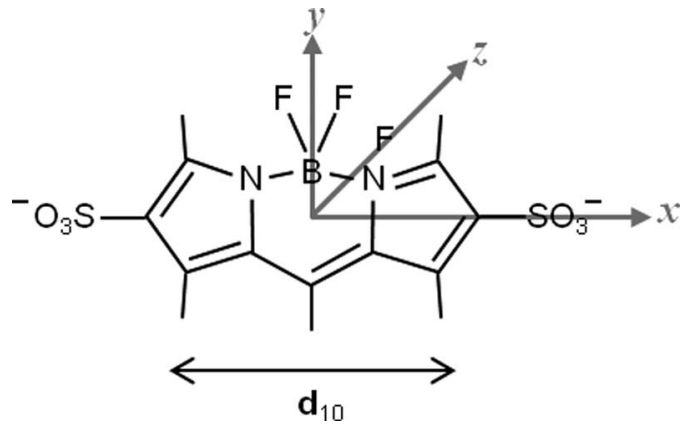

FIG. 1. Molecular structure of the doubly deprotonated pyrromethene-556 dianion $\left(\mathrm{PM}^{2-}\right)$ with the Cartesian reference frame defined. Also shown is the direction of the transition dipole moment, $\mathbf{d}_{10}$, for excitation from $S_{0}$ to $S_{1}$.

reinterpretation of the temporal population dynamics following electronic excitation.

To investigate the effect of the RCB on the dynamics of PEs, we use doubly deprotonated pyrromethene-556 ( $\mathrm{PM}^{2-}$, Fig. 1) ${ }^{24,25}$ It is commonly used as a laser dye and primarily consists of a central boron-dipyrromethene (BODIPY) chromophore, on which the highest occupied molecular orbital (HOMO) of the dianion is located, and two terminal $\mathrm{SO}_{3}{ }^{-}$ groups carrying the excess charges. Numerous studies have investigated the BODIPY chromophore, which has a strong $\mathrm{S}_{1} \leftarrow \mathrm{S}_{0}$ transition centered at $498 \mathrm{~nm}$ (in methanol) and a transition dipole moment vector as indicated in Fig. $1 .^{26-28}$ The electron affinity of $\mathrm{PM}^{2-}$ in the gas-phase has been measured as $1.6 \mathrm{eV}$, making the $\mathrm{S}_{1}$ excited state formally unbound with respect to electron loss. ${ }^{17}$ This state is however located below the RCB but above the $\mathrm{D}_{0}$ monoanion ground state, such that the $S_{1}$ state is meta-stable. The choice of $\mathrm{PM}^{2-}$ is driven by a number of factors. First, the optically bright $\mathrm{S}_{1}$ state decays predominantly by fluorescence in solution $(\Phi=0.92$ in methanol $),{ }^{24,29}$ such that internal conversion (IC) or inter-system crossing are very minor decay pathways in the condensed phase. The observed $S_{1}$ lifetime in the gasphase is significantly faster ${ }^{17}$ than the solution phase fluorescence lifetime $\left(\sim 5 \mathrm{~ns}\right.$ in methanol $\left.{ }^{26,29}\right)$, implying that fluorescence plays a negligible role in the decay of isolated excited $\mathrm{PM}^{2-}$. Second, the rigid structural backbone of $\mathrm{PM}^{2-}$ does not change dramatically upon excitation. Hence, changes in the location of the charged sulfate groups relative to the BODIPY backbone can effectively be neglected. Third, the location of the sulfate groups, and therefore the excess charges, is very well defined with respect to the transition dipole moment of the $\mathrm{S}_{1} \leftarrow \mathrm{S}_{0}$ transition.

In this paper we present results of nanosecond and femtosecond single-color PE imaging as well as pump-probe time-resolved PE imaging and discuss in detail the observed PE spectra and anisotropy following resonant and nonresonant excitation.

\section{METHODS}

\section{A. Experiment}

Isolated $\mathrm{PM}^{2-}$ was produced via an electrospray ionization source and investigated using PE imaging. ${ }^{30-32}$ The experimental setup has been described previously, and only details pertaining to the current experiment are given. ${ }^{33,34}$ Ions were produced via electrospraying of a $1 \mathrm{mM}$ solution of the PM disodium salt (Exciton) in methanol at approximately $-2.5 \mathrm{kV}$. Anions were introduced into vacuum by a desolvation capillary and accumulated in a home-built ring electrode ion trap. Stored ions were then ejected at $200 \mathrm{~Hz}$ into a collinear time-of-flight mass spectrometer. An ion packet of mass-selected $\mathrm{PM}^{2-}$ was intersected by laser pulses at the center of a velocity-map imaging arrangement ${ }^{35}$ and PEs collected on a dual multi-channel plate detector with a phosphor anode. Raw PE images were captured with a CCD camera and deconvoluted using the polar onion-peeling (POP) algorithm developed in our group, ${ }^{36}$ yielding PADs and PE spectra that have been calibrated using the well-known detachment from iodide. The energy resolution is $\sim 5 \%$ of the $e K E$.

Nanosecond laser pulses in the visible spectral region were derived from an Nd:YAG pumped optical parametric oscillator. The nanosecond laser operated at $10 \mathrm{~Hz}$ and consequently the ions in these experiments were also injected into the mass-spectrometer at $10 \mathrm{~Hz}$. Nanosecond pulse energies were attenuated to $\sim 5 \mathrm{~mJ}$ per pulse. The intensity in the interaction region was of the order of $7 \times 10^{6} \mathrm{~W} \mathrm{~cm}^{-2}$.

Femtosecond laser pulses were derived from a commercial Ti:Sapphire oscillator and amplifier system delivering $800 \mathrm{~nm}, 35 \mathrm{fs}$ pulses at $1 \mathrm{kHz}$ repetition rate. Pulses at 266 $\mathrm{nm}$ were generated via frequency tripling using two type I beta-barium borate (BBO) crystals. Pulses across the visible were generated by mixing the output from a commercial optical parametric amplifier with remaining $800 \mathrm{~nm}$ fundamental light. Typical light intensities in the interaction region were of the order of $1 \times 10^{10} \mathrm{~W} \mathrm{~cm}^{-2}$, with a crosscorrelation between $510 \mathrm{~nm}$ and the $800 \mathrm{~nm}$ fundamental of around $\sim 130 \mathrm{fs}$, as determined in a separate thin BBO crystal.

\section{B. Theory}

All electronic structure calculations were carried out using the Gaussian09 software package. ${ }^{37}$ The structure of $\mathrm{PM}^{2-}$ was optimized at the B3LYP/6-311++g $(2 d, 2 p)$ level. The vertical detachment energy was evaluated as the difference in energy between the monoanion and dianion electronic configurations in the dianion optimized geometry. Electronic excited state calculations were carried out using time-dependent density functional theory (TD-DFT) at the B3LYP/6-311++ $\mathrm{g}(2 d, 2 p)$ level for both the monoanion and dianion of PM, in the optimized dianion geometry.

The RCB was calculated using the Local Static Approximation model developed by Dreuw and Cederbaum. ${ }^{38}$ The dianion geometry was optimized using density functional theory at the $\mathrm{B} 3 \mathrm{LYP} / 6-311++\mathrm{g}(2 d, 2 p)$ level. RCB calculations were carried out with the $3-21+\mathrm{g} *$ basis set for $2 \mathrm{D}$ scans and with the $6-311++\mathrm{g}(2 d, 2 p)$ basis set for 1D cuts through the potential energy surface. RCB energies were then evaluated as the difference in total energy between the monoanion electronic configuration in the dianion geometry, and the same configuration in the presence of a point charge. The location of the excess point charge (representing the departing PE) was scanned systematically to build up a 1D or 2D RCB surface. In order to avoid effects of the point charge on the 
molecular charge distribution (polarization effects), a small $-0.01 e$ charge was used and the calculated energy differences scaled accordingly. ${ }^{38}$

\section{RESULTS}

\section{A. Single-color photoelectron spectra}

Single-color PE spectra of $\mathrm{PM}^{2-}$ collected with a femtosecond laser pulse are shown in Fig. 2, along with the corresponding reconstructed $\mathrm{PE}$ images (insets). The PE spectrum collected at a photon energy of $4.66 \mathrm{eV}(266 \mathrm{~nm})$ is shown in Fig. 2(a) and shows two distinctive features: a small broad peak centered at an electron kinetic energy $(e K E)=1.6 \mathrm{eV}$ and a higher energy feature centered at $e K E=2.7 \mathrm{eV}$. The PADs are fitted to the well-known formula ${ }^{39,40}$

$$
I(\theta)=\frac{\sigma}{4 \pi} \sum_{2 n} \beta_{n} P_{n}(\cos \theta),
$$

where $\sigma$ is the photo-detachment cross section; $P_{n}(\cos \theta)$ and $\beta_{n}$ are the $n$th order Legendre polynomial and anisotropy parameter, respectively; and $n$ is an even integer corresponding to the number of photons absorbed in the detachment process. For one photon detachment, $n=1$ so that only $\beta_{2}$ is required to fully describe the PADs (note that $\beta_{0}=1$ ). For a two-photon detachment, $n=2$ and $\beta_{4}$ is additionally required to describe the PADs. The fitting is performed in the POP de-

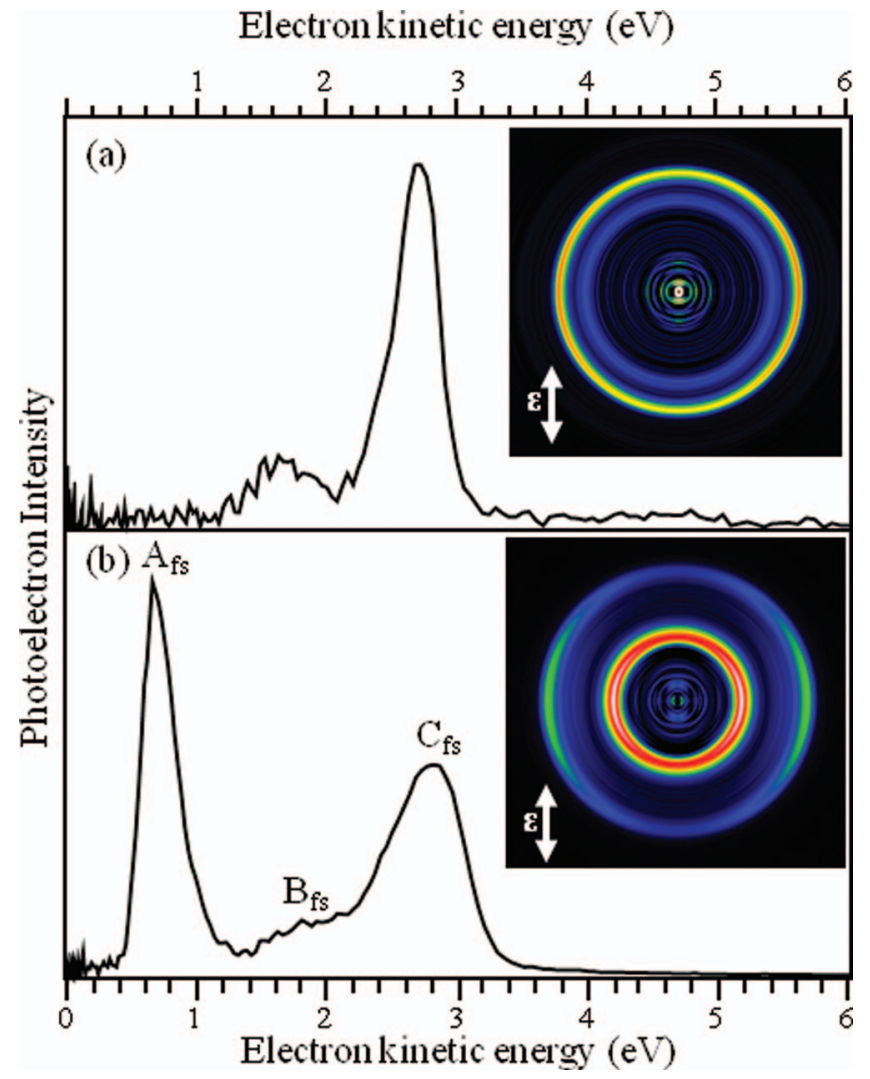

FIG. 2. Photoelectron spectra of $\mathrm{PM}^{2-}$ taken at (a) $4.66 \mathrm{eV}$ and (b) $2.43 \mathrm{eV}$. Insets show the respective reconstructed photoelectron images, in which $\varepsilon$ indicates the polarization direction of the laser field. Reprinted with permission from D. A. Horke, A. S. Chatterley, and J. R. R. Verlet, J. Phys. Chem. Lett. 3, 834 (2012). Copyright 2012 American Chemical Society. convolution routine. ${ }^{36}$ The measured $\mathrm{PE}$ anisotropy parameter for the feature at $e K E=1.6 \mathrm{eV}$ is $\beta_{2}=0.0 \pm 0.2$, while that at $e K E=2.7 \mathrm{eV}$ has $\beta_{2}=-0.12 \pm 0.05$.

The femtosecond PE spectrum collected at a photon energy of $2.43 \mathrm{eV}(510 \mathrm{~nm})$ is shown in Fig. 2(b). At this energy, the photon is resonant with the $\mathrm{S}_{1} \leftarrow \mathrm{S}_{0}$ transition. Three distinct features are observed. Feature $\mathrm{A}_{\mathrm{fs}}$ is a sharp and asymmetrically shaped peak centered around $e K E=0.7 \mathrm{eV}$, feature $\mathrm{B}_{\mathrm{fs}}$ is weaker and centered at $e K E \sim 1.6 \mathrm{eV}$, and feature $\mathrm{C}_{\mathrm{fs}}$ is centered at $e K E=2.8 \mathrm{eV}$. Given that feature $\mathrm{C}_{\mathrm{fs}}$ is at a higher $e K E$ than the photon energy, this feature arises from a twophoton process. Further confirmation for this assignment can be gained from a comparison between the $4.66 \mathrm{eV}$ and 2.43 $\mathrm{eV}$ PE spectra. The similarity in spectral shape and peak positions of features $\mathrm{B}_{\mathrm{fs}}$ and $\mathrm{C}_{\mathrm{fs}}$, compared with the corresponding features in the UV PE spectrum, indicate that both features are arising from a 2-photon process. The large feature in the $4.66 \mathrm{eV}$ PE spectrum therefore comes from a single photon process. The intensity of the PE features in the $2.43 \mathrm{eV}$ $\mathrm{PE}$ spectrum can be ascribed to the fact that the 2-photon PE features (features $B_{f s}$ and $C_{f s}$ ) are resonance enhanced by the $\mathrm{S}_{1} \leftarrow \mathrm{S}_{0}$ transition. Feature $\mathrm{A}_{\mathrm{fs}}$ in Fig. 2(b) is not reproduced in the $4.66 \mathrm{eV}$ PE spectrum. The measured PE anisotropy parameters for features $\mathrm{A}_{\mathrm{fs}}, \mathrm{B}_{\mathrm{fs}}$, and $\mathrm{C}_{\mathrm{fs}}$ are: $\beta_{2}=-0.15 \pm 0.08$; $\beta_{2}=-0.6 \pm 0.1$; and $\beta_{2}=-0.56 \pm 0.05$, respectively. The higher order parameters for $\mathrm{B}_{\mathrm{fs}}$ and $\mathrm{C}_{\mathrm{fs}}$ are $\beta_{4}=0.11 \pm 0.13$ and $\beta_{4}=0.02 \pm 0.05$, respectively, which are essentially zero within experimental error.

Femtosecond PE spectra collected at several photon energies resonant with the $S_{1} \leftarrow S_{0}$ transition are shown in Fig. 3(a), with detachment energies ranging from $2.58 \mathrm{eV}$ $(479 \mathrm{~nm})$ to $2.30 \mathrm{eV}(539 \mathrm{~nm})$. All spectra are similar in appearance to the PE spectrum taken at $2.43 \mathrm{eV}$ with features $\mathrm{A}_{\mathrm{fs}}$ and $\mathrm{C}_{\mathrm{fs}}$ clearly distinguishable. The weaker feature $\mathrm{B}_{\mathrm{fs}}$ is not as well reproduced because of the lower statistics in these spectra. As the photon energy increases, the intensity of feature $\mathrm{C}_{\mathrm{fs}}$ increases relative to feature $\mathrm{A}_{\mathrm{fs}}$. Moreover, feature $A_{f s}$ shows no spectral shift with increasing photon energy. This is in contrast to feature $\mathrm{C}_{\mathrm{fs}}$, which shifts to higher $e K E$ as the photon energy increases, in agreement with the expected dependency of a vertical 2-photon photodetachment transition.

PE spectra of $\mathrm{PM}^{2-}$ collected with nanosecond laser pulses are shown in Fig. 3(b), for various photon energies ranging from $2.23 \mathrm{eV}(540 \mathrm{~nm})$ to $2.70 \mathrm{eV}(460 \mathrm{~nm})$. All spectra show three distinctive features. The first is an isotropic peak, feature $\mathrm{D}_{\mathrm{ns}}$, at very low $e K E$. A second broad feature centered at $e K E=1.3 \mathrm{eV}$ is seen in the PE spectra taken at all detachment energies. This feature exhibits slightly negative anisotropies, with $\beta_{2}=-0.17 \pm 0.09$ at $2.58 \mathrm{eV}(480 \mathrm{~nm})$. We label this feature $A_{n s}$ for reasons that will become apparent below. Finally, at higher $e K E$ a broad feature between 2 and $3.5 \mathrm{eV}$ can be discerned that shifts to higher $e K E$ with increasing photon energy. This feature gains intensity relative to the other features as the photon energy is increased. Based on the $e K E$ of this feature, it is safe to assign this feature to a resonance-enhanced 2-photon detachment process as discussed above for femtosecond excitation. Hence, we label this feature $C_{n s}$. It is significantly more anisotropic than 


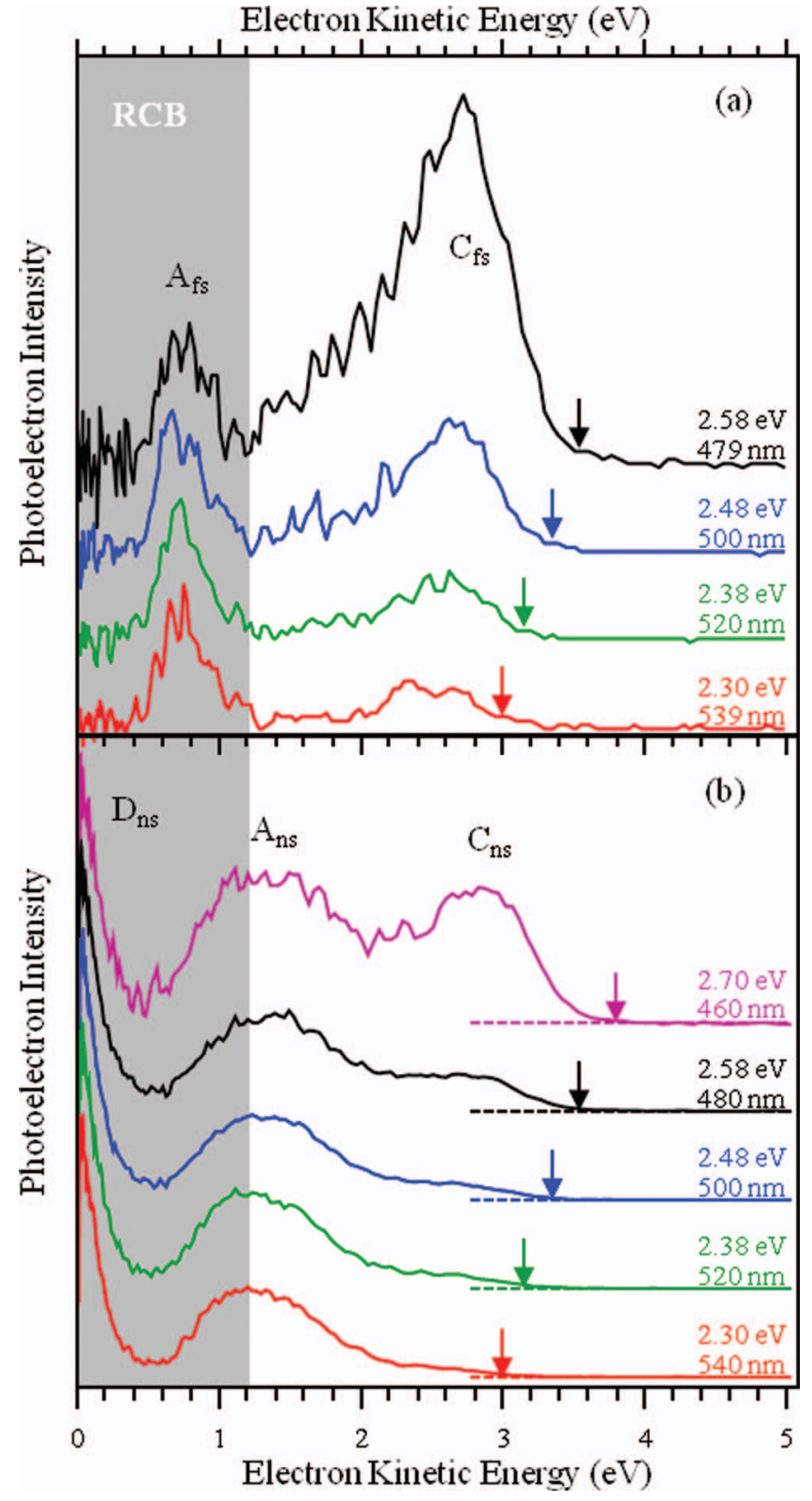

FIG. 3. Photoelectron spectra of $\mathrm{PM}^{2-}$ taken with (a) femtosecond pulses and (b) nanosecond pulses over a range of photon energies as indicated. Downward vertical arrows show the maximum kinetic energy expected for a 2-photon transition at the given photon energy. The grey shading represents the spectral region where the RCB prevents direct electron detachment.

feature $\mathrm{A}_{\mathrm{ns}}$, with typical values for $\beta_{2}=-0.49 \pm 0.08\left(\beta_{4}\right.$ $=0.02 \pm 0.12)$ at $480 \mathrm{~nm}$.

\section{B. Time-resolved photoelectron spectra}

Time-resolved dynamics of the $S_{1}$ dianion excited state were investigated at various pump energies $(2.43,2.34$, and $2.30 \mathrm{eV}$, corresponding to wavelengths of 510,530 , and 539 $\mathrm{nm}$, respectively) and probed at $1.55 \mathrm{eV}(800 \mathrm{~nm})$. Excitation at different pump energies leads to different amounts of internal energy within the $S_{1}$ excited state. Fig. 4(a) shows the PE spectrum when the pump and probe pulses are temporally overlapped, $t=t_{0}$. The probe alone produces no photoelectrons so that pump-probe spectra are directly comparable to
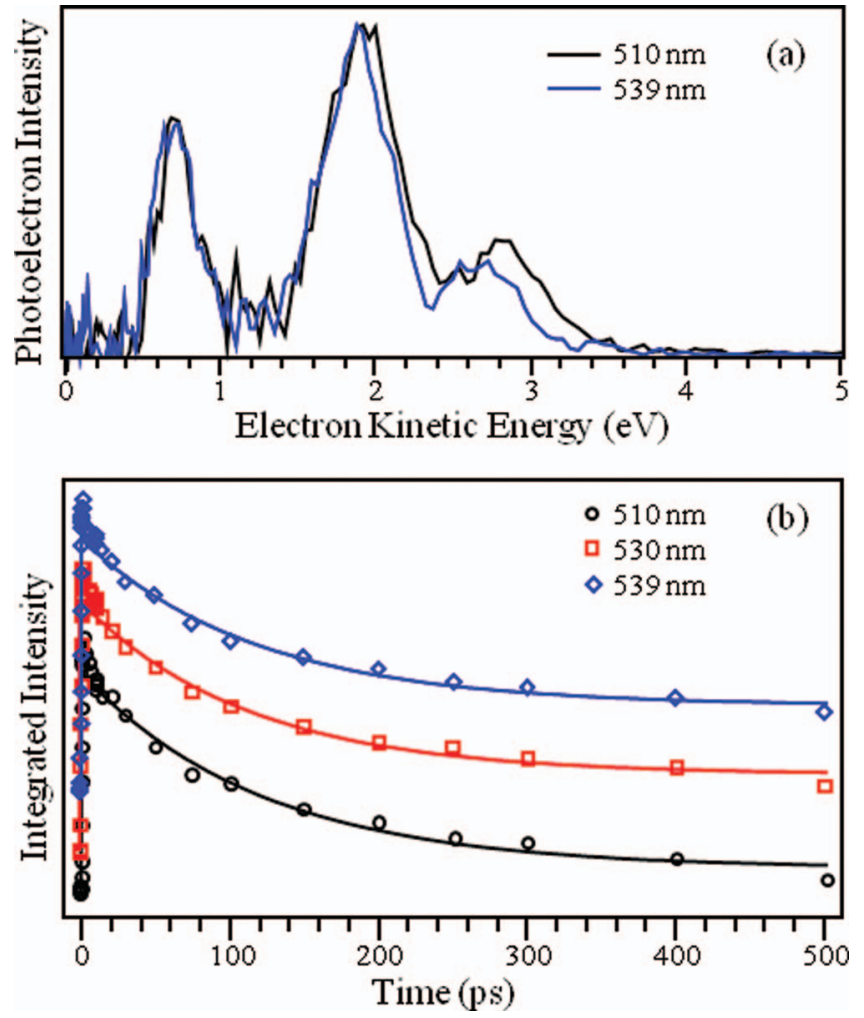

FIG. 4. (a) Time-resolved photoelectron spectra of $\mathrm{PM}^{2-}$ at $t=t_{0}$ taken at two pump photon energies. (b) Integrated photoelectron signal of the pumpprobe feature around $e K E \sim 2 \mathrm{eV}$ as a function of pump-probe delay at the photon energies indicated. Lines are fits to a single exponential decay.

the one-color (at $2.43 \mathrm{eV}$ ) PE spectrum in Fig. 2(b), where any new features must arise from a pump-probe process. A new PE feature is clearly observed between the pump-only features $\mathrm{A}_{\mathrm{fs}}$ and $\mathrm{C}_{\mathrm{fs}}$. Two representative PE spectra taken at $t_{0}$ are shown in Fig. 4(a) for pump energies of $2.43 \mathrm{eV}$ (510 $\mathrm{nm})$ and $2.30 \mathrm{eV}(539 \mathrm{~nm})$. The two spectra show that the peak that can be assigned to feature $A_{\mathrm{fs}}$ does not shift with increasing pump photon energy. In contrast, a spectral blueshift is observed for the pump-probe feature as the photon energy is increased. Similarly, a blueshift in the resonance-enhanced 2-photon feature $\mathrm{C}_{\mathrm{fs}}$ can be seen, which amounts to $\sim 0.25 \mathrm{eV}$, consistent with a 2-photon process.

The dynamics were investigated by scanning the pumpprobe delay, $t$, and integrating over the observed pump-probe $\mathrm{PE}$ feature as a function of $t$. The resulting dynamics traces are shown in Fig. 4(b) for the three pump photon energies used. The integrated PE intensities have been fitted with a single exponential decay, convoluted with a Gaussian instrument response function to account for the finite cross-correlation of pump and probe pulses. ${ }^{41}$ This yields excited state lifetimes of 121,112 , and $117 \pm 10$ ps for pump wavelengths 510 , 530 , and $539 \mathrm{~nm}$, respectively. All three lifetimes are identical within the error margins.

\section{DISCUSSION}

\section{A. Non-resonant femtosecond photoelectron spectra}

The high-energy peak in the $4.66 \mathrm{eV}$ PE spectrum arises from single photon detachment, hence the photon energy must 
be greater than the adiabatic binding energy plus the repulsive Coulomb barrier (RCB) ${ }^{10}$ In such a case, the asymptotic eKE can be related to the intrinsic energetics of the system in the usual fashion. Here the high energy peak is located at $e K E$ $=2.7 \mathrm{eV}$, such that the vertical binding energy is $2.0 \mathrm{eV}$. Extrapolation of the high-energy side of this feature yields an adiabatic detachment energy that is approximately $1.6 \mathrm{eV}$.

To gain support for our assignment and to identify the final states accessed in the radical anion, we use DFT and TD-DFT calculations as described above. We calculate a vertical energy gap from the $S_{0}$ to the $D_{0}$ of $1.95 \mathrm{eV}$, while the adiabatic energy gap is evaluated to be $1.79 \mathrm{eV}$. This is in agreement with our measurements. The $\mathrm{D}_{0}$ state corresponds to removal of an electron from the $\pi$-system of the BODIPY chromophore, and hence photodetachment from the $\mathrm{S}_{0}$ is expected to lead to this electronic state of the anion.

Given the energy gap between the feature at $e K E$ $=2.7 \mathrm{eV}$ and the weaker peak at $1.6 \mathrm{eV}$, the latter most probably arises from the formation of an excited state in the radical anion following electron detachment. An excited state of the anion is calculated at $1.2 \mathrm{eV}$, corresponding primarily to excitation from the HOMO -3 to the HOMO of the anion. This state is weakly correlating with both the $S_{0}$ and $S_{1}$ state of the dianion, providing some support for this assignment.

For $e K E<1.1 \mathrm{eV}$, no PE signal is observed, despite the existence of several higher lying $\mathrm{D}_{n}$ excited states of the monoanion that can be accessed according to our calculations. However, a low-energy cut-off in PE spectra of polyanions is expected because of the RCB, below which no PE can be emitted. Based on the $4.66 \mathrm{eV}$ PE spectrum in Fig. 2(a), we estimate the RCB to be $1.1 \mathrm{eV}$ high. This could indicate that a large fraction of the weak feature at $e K E=1.6 \mathrm{eV}$ cannot be observed because of the proximity of the RCB. Based on the above considerations, we can construct an energy level diagram as shown in Fig. 5.

While the RCB in dianions has been shown to strongly influence the direction of outgoing PEs, guiding them away from the remaining negative charges, ${ }^{17,19,20}$ the observed PADs in the $4.66 \mathrm{eV}$ PE spectrum are nearly isotropic. This implies that the differential photodetachment cross-section for $\mathrm{PM}^{2-}$ is almost isotropic, leading to an equal probabil-

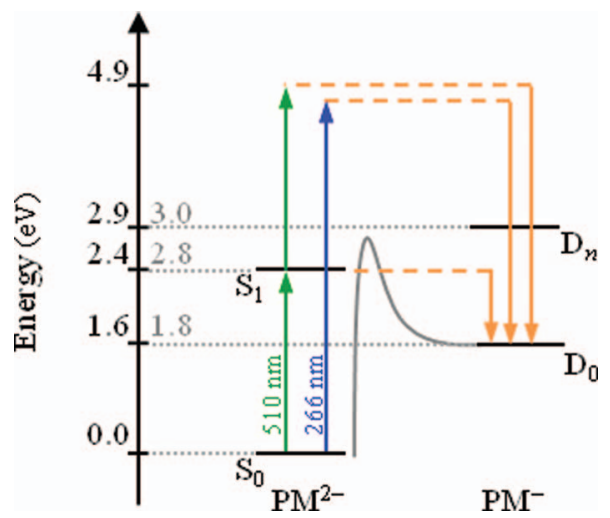

FIG. 5. Energy level diagram of $\mathrm{PM}^{2-}$. Black quantities indicate those determined by photoelectron spectroscopy, while grey quantities are those determined from DFT/TD-DFT calculations. Red downward arrows represent the kinetic energy of emitted photoelectrons. ity of detachment from any molecular orientation in space. Hence, there is no connection between the laboratory and molecular frame of reference, rendering the inherent molecular frame guiding of the PE by the RCB impossible to observe using this scheme. If instead the differential photodetachment cross-section is strongly peaking along a specific molecular orientation, ${ }^{42}$ then the influence of the RCB on the outgoing PE can be observed as shown by the Wang group. ${ }^{19,20}$ However, with no prior knowledge of the detailed differential photodetachment cross-section, the degree of observed anisotropy is convoluted with this unknown and so can be difficult to interpret. To overcome this, the laboratory and molecular frames can be connected through molecular alignment, ${ }^{43,44}$ as discussed below.

\section{B. Resonant femtosecond photoelectron spectra and angular distributions}

The $2.43 \mathrm{eV}$ PE spectrum shown in Fig. 2(b) shows some similarities with the PE spectrum at $4.66 \mathrm{eV}$. Features $\mathrm{B}_{\mathrm{fs}}$ and $\mathrm{C}_{\mathrm{fs}}$ are analogous to those observed following 1-photon detachment at $4.66 \mathrm{eV}$ (Fig. 2(a)), but now proceed via the $S_{1}$ state in a resonance-enhanced 2-photon detachment scheme. However, in the $2.43 \mathrm{eV}$ PE spectrum an additional feature $\left(\mathrm{A}_{\mathrm{fs}}\right)$ can be seen at $e K E=0.7 \mathrm{eV}$, below the estimated $1.1 \mathrm{eV}$ RCB height. In direct photodetachment, PE emission below the RCB is not possible. However, because the $2.43 \mathrm{eV}$ photon is resonant with the $S_{1} \leftarrow S_{0}$ transition, population will be transferred to the $S_{1}$ state of the dianion, where it is trapped by the potential barrier to photodetachment. Given that the $S_{1}$ state in $\mathrm{PM}^{2-}$ is a highly fluorescent state in solution, ${ }^{26,27,29}$ it is not unreasonable to expect the lifetime of the $S_{1}$ state in the gas-phase to be sufficiently long to enable tunneling through the RCB, as has previously been observed in other dianion systems. ${ }^{5,21}$ We therefore assign feature $\mathrm{A}_{\mathrm{fs}}$ to a tunneling process through the RCB from the $S_{1}$ excited state, leaving the anion in the $\mathrm{D}_{0}$ ground state and producing a $\mathrm{PE}$ with $e K E$ significantly below the height of the RCB. Note that such a process is allowed according to Koopmans' correlations in $\mathrm{PM}^{2-}$. This tunneling feature is present at all employed wavelengths in Fig. 3(a), however no shift of the $e K E$ with increasing photon energy is observed. This has previously been explained with a strongly adiabatic mechanism for tunneling through the $\mathrm{RCB}$, which conserves excess internal energy during the tunneling process, leading to emission of electrons with constant $e K E .^{21}$ This is the situation encountered here and tunneling through the RCB is essentially a diagonal process.

All the resonant femtosecond PE spectra in Fig. 3(a) furthermore show a broad feature $\mathrm{C}_{\mathrm{fs}}$ arising from a resonant 2-photon detachment process. The relative intensities of features $\mathrm{A}_{\mathrm{fs}}$ and $\mathrm{C}_{\mathrm{fs}}$ vary with increasing photon energy, with $\mathrm{C}_{\mathrm{fs}}$ becoming more intense relative to $\mathrm{A}_{\mathrm{fs}}$. We can tentatively explain this by the presence of a higher lying electronic state which can be accessed using two-photons. Evidence for this excited state can be seen in the solution phase absorption spectrum, which indicates that there is an absorption maximum at $\sim 240 \mathrm{~nm} .{ }^{24,27}$ Hence, as the photon energy is tuned to the blue, the second photon becomes more resonant with 
excitation of the $S_{1}$ to this higher lying state, increasing the detachment probability.

Comparison of the anisotropy of the features observed in the direct detachment at $4.66 \mathrm{eV}$ with those observed following resonance-enhancement reveals significant differences. Both features $\mathrm{B}_{\mathrm{fs}}$ and $\mathrm{C}_{\mathrm{fs}}$ are observed to be strongly anisotropic following 2-photon detachment, and peaking in the direction perpendicular to the polarization vector of the laser, $\boldsymbol{\varepsilon}$, as indicated in the inset images in Fig. 2. This anisotropy can be traced to the influence of the RCB on the outgoing PE. ${ }^{17}$ The probability of exciting the $S_{1} \leftarrow S_{0}$ transition scales as a function of $\cos ^{2} \theta$, where $\theta$ denotes the angle between the resonant electric field (laser polarization), $\boldsymbol{\varepsilon}$, and the direction of the transition dipole moment, $\mathbf{d}_{\mathbf{1 0}}$. The $\mathbf{d}_{\mathbf{1 0}}$ direction is shown in Fig. 1 and resonant excitation therefore leads to an ensemble of $\mathrm{PM}^{2-}$ molecules excited in their $\mathrm{S}_{1}$ state that have their $x$-axis predominantly aligned with $\varepsilon$. Absorption of a second photon then only probes the ensemble of $\mathrm{S}_{1}$ excited molecules. As the laser field is only $\sim 100 \mathrm{fs}$ in duration, the $S_{1}$ excited $\mathrm{PM}^{2-}$ molecules are essentially frozen in space and the photoemission will occur from an aligned sample. Photodetachment from the $S_{1}$ state leads to the removal of an electron from the $\pi$ system of the BODIPY chromophore and, according to our DFT calculations, the resulting system with a hole on the BODIPY group and charged terminal sulfonates, is the lowest energy state, $\mathrm{D}_{0}$. Given that there is a hole on the central chromophore and two negative charges on the $\mathrm{SO}_{3}$ groups, one might intuitively expect the $\mathrm{PE}$ angular distribution to peak in the direction perpendicular to $\boldsymbol{\varepsilon}$. This is what is observed and it is in stark contrast to the PE angular distribution of the similar feature in the single photon $4.66 \mathrm{eV} \mathrm{PE}$ spectrum, which is essentially isotropic. This clearly demonstrates the importance of establishing a connection between the laboratory and molecular frames of reference.

To gain additional insight into these qualitative arguments, a 2D cut through the RCB surface has been calculated. Shown in Fig. 6(a) is a contour map of the RCB in the $x y$ plane of the molecule (where the Cartesian axes are shown in Fig. 1). Additionally, in Fig. 6(b) representative cuts through the RCB from the center of the molecule along each Cartesian coordinate are shown. As expected, the RCB is largest close to the terminal charges on the $\mathrm{SO}_{3}{ }^{-}$groups and falls off towards the center of the molecule. The maximum of the $\mathrm{RCB}$ is around $5 \mathrm{eV}$ and it should be noted that free rotation around the $\mathrm{C}-\mathrm{S}$ bond will smear out the local maxima identified at each $\mathrm{O}$ site. This explains why the cut along the $x$-axis is lower than this $5 \mathrm{eV} \mathrm{RCB} \mathrm{maximum.} \mathrm{Along} \mathrm{the} y$-axis, the $\mathrm{RCB}$ is asymmetric around the center of the molecule. A local maximum $(3.2 \mathrm{eV})$ is observed near the electron rich boron center on the BODIPY chromophore. Along the positive $y$ direction, the lowest point in the RCB can be identified. The energy of this saddle point is $1.1 \mathrm{eV}$, in excellent agreement with the $4.66 \mathrm{eV} \mathrm{PE}$ spectrum $e K E$ cut-off. It is furthermore consistent with the inability to directly detach an electron at $2.43 \mathrm{eV}$, given the adiabatic binding energy of $1.6 \mathrm{eV}$.

Following resonance-enhanced 2-photon detachment at $2.43 \mathrm{eV}$, the PE will have an asymptotic eKE of about $3.1 \mathrm{eV}$. Comparing this with the calculated RCB surface in Fig. 6(a)
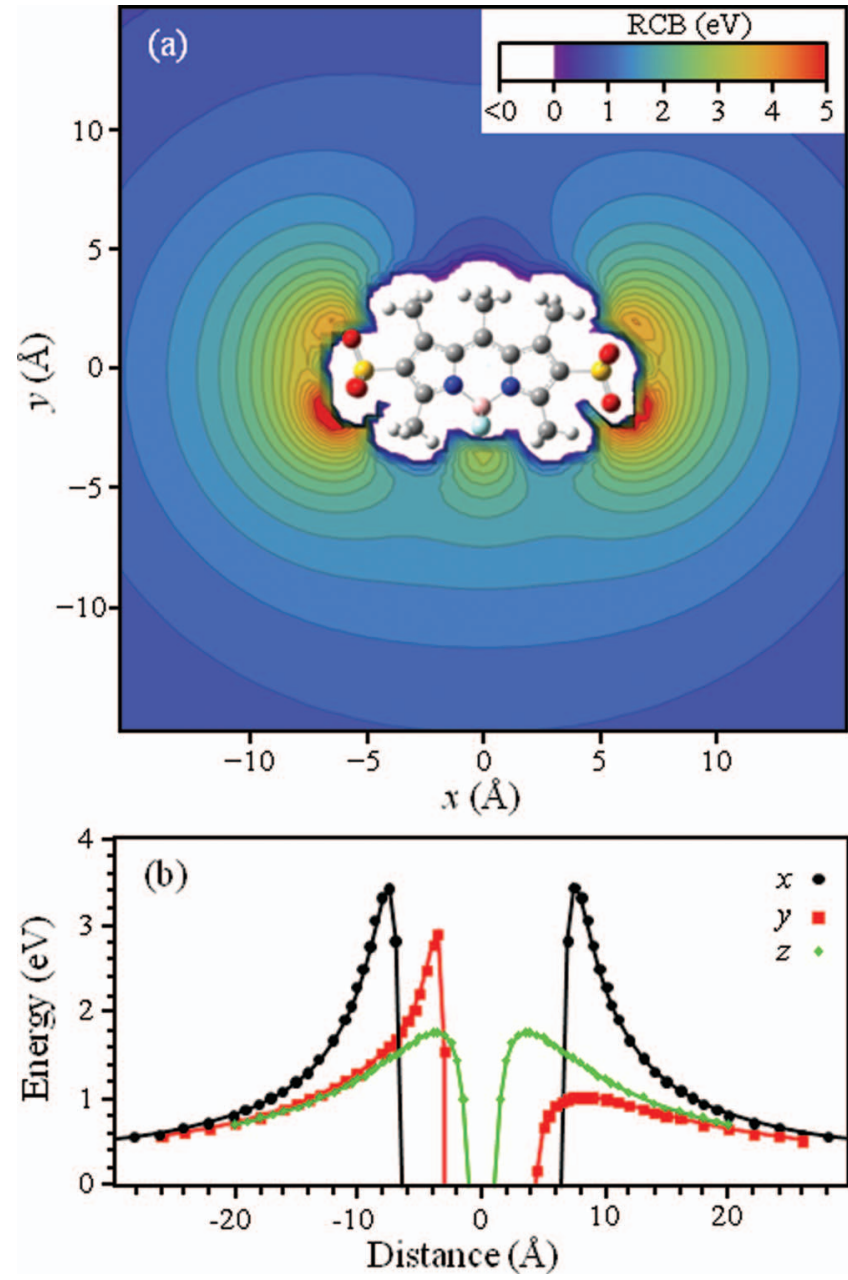

FIG. 6. Calculated repulsive Coulomb barrier for $\mathrm{PM}^{-}+\mathrm{e}^{-}$. (a) $2 \mathrm{D} \mathrm{RCB}$ surface along the $x-y$ plane $(z=0)$. (b) $1 \mathrm{D}$ cuts through the RCB originating at the center of the molecule, along representative Cartesian coordinates as defined in Fig. 1.

allows one to rationalize the observed PADs. The available kinetic energy is not sufficient for the electron to leave in the $x$ direction (along the $\mathrm{SO}_{3}{ }^{-}$groups), instead electrons will primarily be ejected along the $+y$-direction (or $-y$-direction as $\mathrm{PM}^{2-}$ is only $1 \mathrm{D}$ aligned). The lowest saddle point at $1.1 \mathrm{eV}$ is quite shallow along the $x$-direction, suggesting that electrons could have quite a spread in angular emission. Hence we do not observe extreme anisotropy parameters of $\beta_{2}=-1$. The barrier indicated by the cut along the negative $y$-direction shows a RCB at the boron site of $\sim 3.2 \mathrm{eV}$, which is just above the electron energy of feature $\mathrm{C}_{\mathrm{fs}}$ and so will inhibit detachment along this coordinate. However, either side of the boron, saddle-points in the RCB (at the electro-positive methyl groups) should enable the electron to escape through these gaps as the RCB height here is of the order of $2 \mathrm{eV}$.

Once the PE leaves the molecule it will experience a force due to the RCB, which will guide the electron. As this force is simply the gradient of the RCB, one might expect that the PE will be further guided to be perpendicular to the $x$-coordinate. Such an interpretation is based on classical arguments. Although this is clearly not appropriate to describe the photodetachment process, it does provide a very useful framework 
to qualitatively understand observations and the RCB guiding appears to overcome any inherent anisotropy resulting from the quantum mechanical interference of the outgoing partial waves. It would be of interest to investigate asymptotically the electron emission both using quantum and classical simulations, but this is beyond the scope of the present study. We note that, in principle, interference should be present in the angular coordinate as there is more than one path to reach a given point on the detector. We have made very careful and high-quality measurements in an attempt to observe such features, but were unsuccessful. One possible reason for this is the finite and relatively high temperature of the ions $(\sim 300 \mathrm{~K})$.

No appreciable anisotropy is observed for the feature corresponding to tunneling through the RCB (feature $A_{\mathrm{fs}}$ ), despite this process also relying on resonant excitation to the $S_{1}$ excited state. The key difference between this feature and features $\mathrm{B}_{\mathrm{fs}}$ and $\mathrm{C}_{\mathrm{fs}}$ is that tunneling is not a direct process and so the PE is not created "instantaneously" (i.e., within the laser field). Instead, PE emission occurs once the excited state electron has had time to tunnel through the RCB. From the timeresolved PE dynamics shown in Fig. 4(b), this occurs on a timescale a few orders of magnitude longer than the rotational dynamics. In free space, $\mathrm{PM}^{2-}$ can rotate and the absence of anisotropy then suggests that the initially aligned ensemble of dianions in the $S_{1}$ excited state is no longer aligned in the laboratory frame due to rotational dephasing, resulting in an isotropic emission of electrons. The rotational dynamics can be tracked in real-time through the PAD of the pump-probe feature as we have recently shown. ${ }^{17}$

\section{Excited state dynamics}

Following excitation to the $S_{1}$ state, the relaxation dynamics can be investigated using a delayed probe pulse. Representative PE spectra at $t=t_{0}$ are shown in Fig. 4(a). The pump-probe feature blueshifts with increasing photon energy, as expected for a direct (vertical) detachment process induced by the probe. As a function of time, no noticeable spectral changes are observed, except for a reduction in the intensity of the pump-probe feature. The time-varying intensity of this pump-probe feature is a direct reflection of the $S_{1}$ state population. However, the decay mechanism cannot be unambiguously determined. Given that a tunneling feature $\left(\mathrm{A}_{\mathrm{fs}}\right)$ can be clearly identified, tunneling must be occurring. However, internal conversion and intersystem crossing are in principle also possible. Fluorescence will occur on a nanosecond timescale and may be expected to contribute in a very minor role given the $\sim 120$ ps lifetime. ${ }^{26,29}$ Intersystem crossing is improbable as BODIPY dyes in general are well-known for their very low rate of $S_{1} \rightarrow T_{1}$ conversion. ${ }^{24,25}$ However, it cannot be conclusively ruled out. Finally, internal conversion is possible although it should be noted that the fluorescence quantum yield in solution is very high.

Some indication of the dominant pathway can be discerned by the dynamics of the tunneling feature $\mathrm{A}_{\mathrm{fs}}$. If tunneling was the only (or dominant) decay mechanism, one would expect to observe depletion in the intensity of feature $A_{\mathrm{fs}}$ at $t_{0}$, followed by a recovery that mirrors the decay dynamics of the pump-probe signal. ${ }^{31,45}$ If the dynamics were solely from tunneling, then the integrated PE counts of the depletion should equal that of the pump-probe feature, as observed in the fluorescein dianion. ${ }^{21}$ This is not the case and feature $A_{\mathrm{fs}}$ shows no discernible depletion, although we note that the data for this are quite noisy. It therefore appears that the dominant decay mechanism is internal conversion (or intersystem crossing), rather than electron tunneling. This is surprising because of the dominant fluorescence decay path for PM in solution. The main difference between the isolated and solvated $\mathrm{PM}^{2-}$ is the stabilization of the $\mathrm{SO}_{3}{ }^{-}$groups. Minor environmental effects have been noted on PM in different solvents, but these have been associated with effects on the resonance structures of the BODIPY chromophore and the $\mathrm{SO}_{3}{ }^{-}$groups are essentially spectators. ${ }^{29}$ Nonetheless, the fact that these groups are not solvated in the gas-phase leads to strong Coulomb interactions with the BODIPY chromophore $\pi$-system, which may open up internal conversion pathways from the $S_{1}$ excited state.

As the same pump-probe feature acts as a measure of both internal conversion and tunneling, the observed lifetime is a combination of the individual contributions $\left(k_{\mathrm{obs}}=k_{\mathrm{IC}}\right.$ $+k_{\text {tunnel }}$, where $k=\tau^{-1}$ is the rate constant). The measured $\sim 120 \mathrm{ps}$ is thus a lower bound of the internal conversion lifetime, while the tunneling kinetics will be significantly slower (i.e., $\tau_{\text {tunnel }} \gg 120 \mathrm{ps}$ ).

\section{Effect of pulse duration on photoelectron spectra}

The observed peak at very low $e K E$ in the PE spectra taken with a nanosecond laser of Fig. 3(b), feature $D_{n s}$, is unexpected. As discussed before, the presence of the RCB should inhibit the emission of electrons at such low energy. This "forbidden" region is highlighted in Fig. 3 as a grey shaded area, extending to the lowest RCB height of $1.1 \mathrm{eV}$. Low energy features have been observed before by the Wang group in dicarboxylate dianions. ${ }^{46}$ These were assigned to the dissociative autodetachment from an anion, formed following photodetachment of the parent dianion. Specifically, it was proposed that the parent radical anion can exothermically undergo decarboxylation forming the $\left(\mathrm{CH}_{2}\right)_{n} \mathrm{CO}_{2}{ }^{-}$radical, which can then undergo electron loss, leading to low energy PEs. Part of their assignment was based on the observed structure in the PE spectra at $e K E<0.1 \mathrm{eV}$. We also note that until the use of PE imaging to study the spectroscopy of polyanions (i.e., before $2008^{20}$ ), such low $e K E$ features would not have been observed because of the poor efficiency of magnetic bottle PE spectrometers at low $e K E$.

In the present case, the low energy electrons must also come from a monoanion. However, the origin of the peak is rather different. This can be appreciated immediately by the fact that feature $D_{n s}$ is not seen in the PE spectra taken with femtosecond pulses, even at very low intensities. Moreover, feature $\mathrm{D}_{\mathrm{ns}}$ is structureless and isotropic, decaying exponentially with $e K E$. Exponentially decaying PE spectra peaking at threshold are often indicators for statistical autodetachment or thermionic emission. ${ }^{47-50}$ Taking the above observations 
into account, and the fact that no fragmentation is expected following a single excitation to the $S_{1}$ state, we recognize that the system must be acquiring excessive amounts of internal energy, which can then lead to dissociation and electron loss.

The mechanism by which $\mathrm{PM}^{2-}$ can acquire such high internal energy (be it in electronic or vibrational degrees of freedom) can be reconciled by the fact that the nanosecond laser field remains resonant with the $\mathrm{S}_{1} \leftarrow \mathrm{S}_{0}$ transition for the duration of the pulse width $(\sim 10 \mathrm{~ns})$ and by the fact that the dominant decay pathway is internal conversion, which is occurring on a timescale that is at least one order of magnitude faster than the laser pulse. Thus many photons can be absorbed in a similar vein to IR multiphoton dissociation, but using an electronic rather than a vibronic transition. ${ }^{51,52}$ With each photon cycle, the internal energy is increased (by $\sim 2.5 \mathrm{eV}$ ) and will ultimately lead to a sufficiently high internal energy that unimolecular decay will occur. Given the photon energy, this would require very few cycles. Our proposed picture is in agreement with the statistical nature (exponential decay and isotropy of the PAD) of feature $\mathrm{D}_{\mathrm{ns}}$.

The second feature in the nanosecond PE spectra $\left(\mathrm{A}_{\mathrm{ns}}\right)$, centered at $e K E=1.3 \mathrm{eV}$, appears to show no shift towards higher $e K E$ with increasing photon energy and may be correlated with feature $A_{\mathrm{fs}}$ in the femtosecond $\mathrm{PE}$ spectrum in Figs. 2(b) and 3(a). We have shown previously that the invariance of the peak $e K E$ to detachment photon energy can be attributed to a strongly adiabatic electron tunneling process through the RCB. ${ }^{21}$ This has a propensity to conserve the internal energy of the dianion into the anion as the electron is lost. The fact that features $\mathrm{A}_{\mathrm{fs}}$ and $\mathrm{A}_{\mathrm{ns}}$ do not shift with photon energy over a range of $0.4 \mathrm{eV}$ indicates that a similar behavior may be responsible. However, some clear differences are also apparent between $\mathrm{A}_{\mathrm{fs}}$ and $\mathrm{A}_{\mathrm{ns}}$.

The comparison between the tunneling peak in the femtosecond versus nanosecond experiments shows that the maxima of $\mathrm{A}_{\mathrm{fs}}$ and $\mathrm{A}_{\mathrm{ns}}$ are located at $e K E=0.7 \mathrm{eV}$ and $1.3 \mathrm{eV}$, respectively. Furthermore, the width has increased significantly in $A_{n s}$. This is in contrast to our previous experiments on doubly deprotonated fluorescein dianions, in which femtosecond and nanosecond spectra were identical. ${ }^{21}$ The important difference between these two systems is that, in the fluorescein dianion, the tunneling lifetime is of the order of $1 \mathrm{ps}$, and therefore no photon cycling is possible. A similar observation has recently been reported in a tetra-anion. ${ }^{22}$ When using femtosecond pulses, multiple photon cycling is also not possible, irrespective of tunneling life, and the PE spectra taken at different energies reveal no shift in feature $A_{\mathrm{fs}}$, while feature $\mathrm{C}_{\mathrm{fs}}$ clearly does shift (Fig. 3(a)).

The broadening observed in $\mathrm{PM}^{2-}$ is presumably a consequence of the internal heating induced by photon cycling. The lowest $e K E$ of the tunneling feature is the same in both the femto- and nanosecond PE spectra and the broadening is only observed towards higher $e K E$. Moreover, the maximum of feature $\mathrm{A}_{\mathrm{ns}}$ is at $e K E \sim 1.3 \mathrm{eV}$, which is close to but just above the RCB height at $1.1 \mathrm{eV}$. This suggests that a significant fraction of the "tunneling" feature may contain contributions from detachment above the lowest RCB, enabled by the high internal energy. This would appear to contradict the strongly adiabatic tunneling picture observed in the fluo-

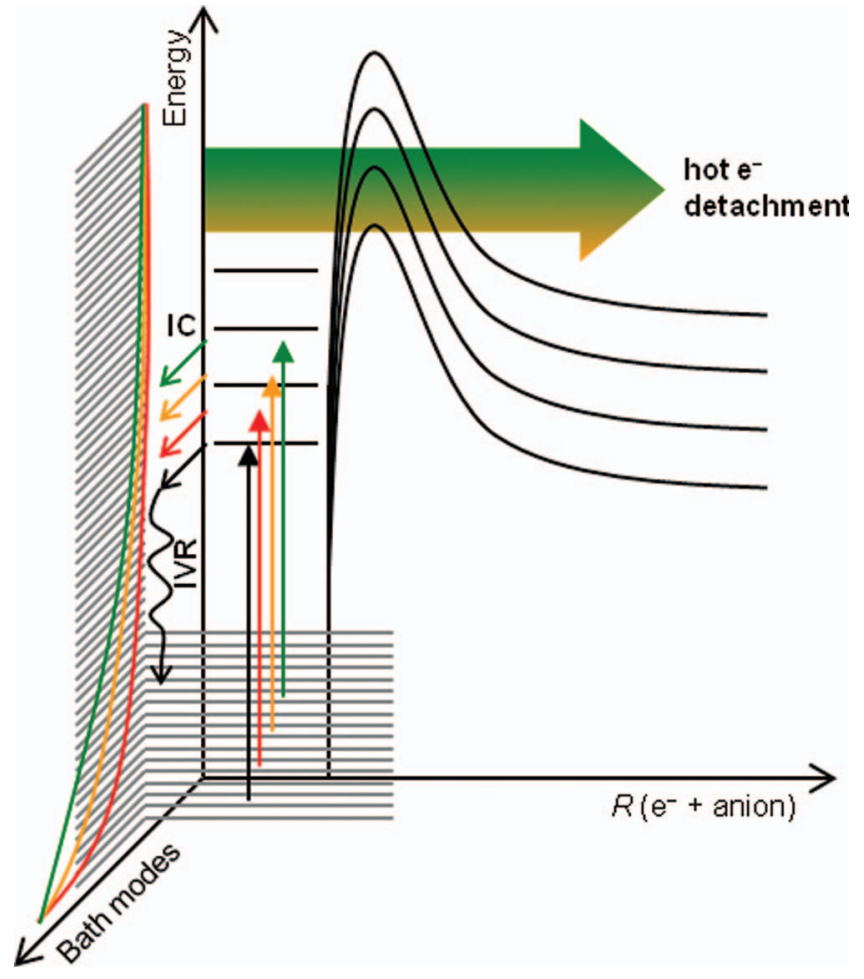

FIG. 7. Schematic diagram explaining the photon-cycling scheme observed in $\mathrm{PM}^{2-}$ that ultimately leads to photoelectrons being emitted nonadiabatically just above the lowest RCB. Each color represents a successive cycle and the internal temperature (represented by population profiles of the bath modes) is shown to increase at every cycle by $\sim 2.5 \mathrm{eV}$. After a small number of cycles, electrons can escape either from the $S_{1}$ excited state or the $\mathrm{S}_{0}$ ground state, by accessing the continuum above the lowest RCB.

rescein dianion. However, the timescales of the electron loss in the present case are much longer than in the fluorescein dianion. Complete intra-molecular vibrational redistribution (IVR) of the internal energy in $\mathrm{PM}^{2-}$ may be expected and so modes that lead to electron emission over the lowest barrier can be statistically sampled. Hence, non-adiabatic tunneling becomes possible and probably dominates in this long-time regime. Such a picture is consistent with both spectral broadening of feature $A_{n s}$ relative to $A_{f s}$ and a blueshift towards the lowest point of the RCB, which is close to the maximum observed in feature $A_{n s}$.

This mechanistic picture is outlined in Fig. 7. Upon excitation, the RCB experienced by the system increases because of the adiabatic tunneling, as discussed in the fluorescein dianion. The system undergoes IVR on the $S_{1}$ excited state. This is followed or in competition with internal conversion back to $\mathrm{S}_{0}$, where additional IVR will occur. The system then absorbs additional photons, instigating the same cascading process. However, now there is already $\sim 2.5 \mathrm{eV}$ of internal energy in the system before photoexcitation. Once in the $S_{1}$ with this additional internal energy content, IVR on the $S_{1}$ excited state could lead to a statistical sampling of the modes that lead to electron loss over the lowest RCB. Alternatively, this non-adiabatic tunneling process is also possible from the ground electronic state $\left(\mathrm{S}_{0}\right)$ following a small number of photon cycles. We have represented this latter scenario in Fig. 7, but experimentally, we cannot determine whether 
feature $A_{n s}$ arises from non-adiabatic tunneling from the $S_{1}$ or $S_{0}$ states. Nevertheless, these statistical views are important as it is through such mechanisms that electron loss and fragmentation can occur for thermally activated polyanions in tandem mass-spectrometry experiments. ${ }^{53,54}$

In addition to this statistical emission over the RCB, tunneling may also contribute to the observed feature. The number and shape of RCB surfaces for both the $S_{1}$ and $S_{0}$ states will depend on the extent of internal excitation. Therefore, the relevant RCB surfaces after a photon cycle are likely to be different than those at $300 \mathrm{~K}$. Hence, excitation from a hot $\mathrm{S}_{0}$ ground state (after a photon cycle) will expose the $\mathrm{S}_{1}$ state to different RCB surfaces and this may lead to broadening of the tunneling peak. This may be expected to be particularly important in non-rigid polyanions.

The PE feature attributed to resonance-enhanced 2photon detachment with a nanosecond laser pulse $\left(\mathrm{C}_{\mathrm{ns}}\right)$ exhibits strong anisotropies of the order of $\beta_{2}=-0.5$ at all employed wavelengths. This is similar to those observed for feature $\mathrm{C}_{\mathrm{fs}}$ following femtosecond excitation. In the latter case, the short pulse durations lead to the absorption of the second photon from an aligned ensemble, producing the highly anisotropic distribution of PEs. ${ }^{17}$ This argument does not hold for nanosecond excitation as the second photon is likely to be absorbed significantly later than the typical rotational dephasing time (a few ps). One might therefore expect to observe an isotropic PE distribution.

A possible explanation for the observed anisotropy could be the involvement of a 2-photon resonance, discussed briefly above, due to the additional state seen at $\sim 240 \mathrm{~nm}$ in the absorption spectrum. This resonance could lead to a nonisotropic absorption cross-section for the second photon, leading to a preferential resonance-enhanced process from a particular orientation and hence an anisotropic distribution of PEs. However, we have no experimental evidence to support this except for the relative increase in detachment of feature $\mathrm{C}_{\mathrm{fs}}$ to $\mathrm{A}_{\mathrm{fs}}$ seen in Fig. 3.

\section{CONCLUSION}

We have presented an extensive study of the gaseous dianion $\mathrm{PM}^{2-}$ using $\mathrm{PE}$ imaging with femtosecond and nanosecond laser pulses. The femtosecond measurements confirm the strongly adiabatic nature of electron tunneling through the RCB previously reported. However, this was not the situation observed following nanosecond excitation, where the dominant internal conversion decay channel leads to multiple photon cycling via an electronic transition and the build-up of excessive amounts of internal energy in the system. Within the nanosecond pulse duration, this energy becomes redistributed and eventually samples modes leading to detachment over the lowest RCB. This shifts the observed PE signal towards the lowest energy on the RCB surface. Hence the timescale of the excitation and available decay routes play a crucial role in interpreting PE spectra of dianions.

Using time-resolved photoelectron spectroscopy, the lifetime of the $S_{1}$ excited state was evaluated as $\sim 120$ ps, independent of excitation energy. The observed PE angular distributions can be rationalized with the molecular alignment following an allowed electronic transition. The direction of any outgoing PEs is strongly dependent on the RCB surface, an effect that outcompetes any inherent angular distribution due to partial wave interferences. The RCB was calculated using the Local Static Approximation approach and agrees qualitatively with experimental data.

\section{ACKNOWLEDGMENTS}

We are grateful to the EPSRC laser loan pool for loan of the ns laser system. This work has been supported by the EPSRC (EP/D073472/1) and Durham University. A.S.C. is funded by the Leverhulme Trust. J.R.R.V. thanks the ERC for support.

${ }^{1}$ A. Dreuw and L. S. Cederbaum, Chem. Rev. 102, 181 (2002).

${ }^{2}$ M. K. Scheller, R. N. Compton, and L. S. Cederbaum, Science 270, 1160 (1995).

${ }^{3}$ X.-B. Wang and L.-S. Wang, Annu. Rev. Phys. Chem. 60, 105 (2009).

${ }^{4}$ O. T. Ehrler, J. M. Weber, F. Furche, and M. M. Kappes, Phys. Rev. Lett. 91, 113006 (2003).

${ }^{5}$ O. T. Ehrler, J. P. Yang, A. B. Sugiharto, A. N. Unterreiner, and M. M. Kappes, J. Chem. Phys. 127, 184301 (2007).

${ }^{6}$ K. Matheis, L. Joly, R. Antoine, F. Lepine, C. Bordas, O. T. Ehrler, A. R. Allouche, M. M. Kappes, and P. Dugourd, J. Am. Chem. Soc. 130, 15903 (2008).

${ }^{7}$ J. Simons, J. Phys. Chem. A 112, 6401 (2008).

${ }^{8}$ L. S. Wang, C. F. Ding, X. B. Wang, J. B. Nicholas, and B. Nicholas, Phys. Rev. Lett. 81, 2667 (1998).

${ }^{9}$ X.-B. Wang and L.-S. Wang, Nature (London) 400, 245 (1999).

${ }^{10}$ X. B. Wang, C. F. Ding, and L. S. Wang, Phys. Rev. Lett. 81, 3351 (1998).

${ }^{11}$ R. N. Compton, A. A. Tuinman, C. E. Klots, M. R. Pederson, and D. C. Patton, Phys. Rev. Lett. 78, 4367 (1997).

${ }^{12}$ R. L. Hettich, R. N. Compton, and R. H. Ritchie, Phys. Rev. Lett. 67, 1242 (1991).

${ }^{13}$ C. Jin, R. L. Hettich, R. N. Compton, A. Tuinman, A. Derecskei-Kovacs, D. S. Marynick, and B. I. Dunlap, Phys. Rev. Lett. 73, 2821 (1994).

${ }^{14}$ C. Yannouleas and U. Landman, Chem. Phys. Lett. 210, 437 (1993).

${ }^{15}$ J. M. Weber, I. N. Ioffe, K. M. Berndt, D. Löffler, J. Friedrich, O. T. Ehrler, A. S. Danell, J. H. Parks, and M. M. Kappes, J. Am. Chem. Soc. 126, 8585 (2004).

${ }^{16}$ X.-B. Wang, J. B. Nicholas, and L.-S. Wang, J. Chem. Phys. 113, 653 (2000).

${ }^{17}$ D. A. Horke, A. S. Chatterley, and J. R. R. Verlet, J. Phys. Chem. Lett. 3, 834 (2012)

${ }^{18}$ C.-G. Ning, P. D. Dau, and L.-S. Wang, Phys. Rev. Lett. 105, 263001 (2010).

${ }^{19}$ X.-P. Xing, X.-B. Wang, and L.-S. Wang, J. Phys. Chem. A 113, 945 (2009).

${ }^{20}$ X. P. Xing, X. B. Wang, and L. S. Wang, Phys. Rev. Lett. 101, 083003 (2008).

${ }^{21}$ D. A. Horke, A. S. Chatterley, and J. R. R. Verlet, Phys. Rev. Lett. 108, 083003 (2012).

${ }^{22}$ P. D. Dau H.-T. Liu, J.-P. Yang, M.-O. Winghart, T. J. A. Wolf, A.-N. Unterreiner, P. Weis, Y.-R. Miao, C.-G. Ning, M. M. Kappes, and L.-S. Wang, Phys. Rev. A 85, 064503 (2012).

${ }^{23}$ M.-O. Winghart, J.-P. Yang, M. Kuhn, A.-N. Unterreiner, T. J. A. Wolf, P. D. Dau, H.-T. Liu, D.-L. Huang, W. Klopper, L.-S. Wang, and M. M. Kappes, Phys. Chem. Chem. Phys. 15, 6726 (2013).

${ }^{24}$ S. C. Guggenheimer, J. H. Boyer, K. Thangaraj, M. Shah, M.-L. Soong, and T. G. Paviopoulos, Appl. Opt. 32, 3942 (1993).

${ }^{25}$ T. G. Pavlopoulos, M. Shah, and J. H. Boyer, Opt. Commun. 70, 425 (1989).

${ }^{26}$ Y. Assor, Z. Burshtein, and S. Rosenwaks, Appl. Opt. 37, 4914 (1998).

${ }^{27}$ F. López Arbeloa, J. Bañuelos, V. Martînez, T. Arbeloa, and I. López Arbeloa, Int. Rev. Phys. Chem. 24, 339 (2005).

${ }^{28}$ A. Loudet, and K. Burgess, Chem. Rev. 107, 4891 (2007).

${ }^{29}$ T. López Arbeloa, F. López Arbeloa, and I. López Arbeloa, Phys. Chem. Chem. Phys. 1, 791 (1999).

${ }^{30}$ R. Mabbs, E. R. Grumbling, K. Pichugin, and A. Sanov, Chem. Soc. Rev. 38, 2169 (2009). 
${ }^{31}$ J. R. R. Verlet, Chem. Soc. Rev. 37, 505 (2008).

${ }^{32}$ G. Wu, P. Hockett, and A. Stolow, Phys. Chem. Chem. Phys. 13, 18447 (2011).

${ }^{33}$ J. Lecointre, G. M. Roberts, D. A. Horke, and J. R. R. Verlet, J. Phys. Chem. A 114, 11216 (2010).

${ }^{34}$ D. A. Horke, G. M. Roberts, J. Lecointre, and J. R. R. Verlet, Rev. Sci. Instrum. 83, 063101 (2012).

${ }^{35}$ A. T. J. B. Eppink, and D. H. Parker, Rev. Sci. Instrum. 68, 3477 (1997).

${ }^{36}$ G. M. Roberts, J. L. Nixon, J. Lecointre, E. Wrede, and J. R. R. Verlet, Rev. Sci. Instrum. 80, 053104 (2009).

${ }^{37}$ M. J. Frisch, G. W. Trucks, H. B. Schlegel, G. E. Scuseria, M. A. Robb, J. R. Cheeseman, G. Scalmani, V. Barone, B. Mennucci, G. A. Petersson, H. Nakatsuji, M. Caricato, X. Li, H. P. Hratchian, A. F. Izmaylov, J. Bloino, G. Zheng, J. L. Sonnenberg, M. Hada, M. Ehara, K. Toyota, R. Fukuda, J. Hasegawa, M. Ishida, T. Nakajima, Y. Honda, O. Kitao, H. Nakai, T. Vreven, J. A. Montgomery, Jr., J. E. Peralta, F. Ogliaro, M. Bearpark, J. J. Heyd, E. Brothers, K. N. Kudin, V. N. Staroverov, R. Kobayashi, J. Normand, K. Raghavachari, A. Rendell, J. C. Burant, S. S. Iyengar, J. Tomasi, M. Cossi, N. Rega, J. M. Millam, M. Klene, J. E. Knox, J. B. Cross, V. Bakken, C. Adamo, J. Jaramillo, R. Gomperts, R. E. Stratmann, O. Yazyev, A. J. Austin, R. Cammi, C. Pomelli, J. W. Ochterski, R. L. Martin, K. Morokuma, V. G. Zakrzewski, G. A. Voth, P. Salvador, J. J. Dannenberg, S. Dapprich, A. D. Daniels, Ö. Farkas, J. B. Foresman, J. V. Ortiz, J. Cioslowski, and D. J. Fox, Gaussian 09, Revision A.02, Gaussian, Inc., Wallingford, CT, 2009.

${ }^{38}$ A. Dreuw and L. S. Cederbaum, Phys. Rev. A 63, 049904 (2001).

${ }^{39}$ J. Cooper and R. N. Zare, J. Chem. Phys. 48, 942 (1968).
${ }^{40}$ K. L. Reid, Annu. Rev. Phys. Chem. 54, 397 (2003).

${ }^{41}$ D. A. Horke, and J. R. R. Verlet, Phys. Chem. Chem. Phys. 13, 19546 (2011).

${ }^{42}$ D. Dill, J. Chem. Phys. 65, 1130 (1976).

${ }^{43}$ K. F. Lee, D. M. Villeneuve, P. B. Corkum, A. Stolow, and J. G. Underwood, Phys. Rev. Lett. 97, 173001 (2006).

${ }^{44}$ H. Stapelfeldt and T. Seideman, Rev. Mod. Phys. 75, 543 (2003).

${ }^{45}$ G. M. Roberts, J. Lecointre, D. A. Horke, and J. R. R. Verlet, Phys. Chem. Chem. Phys. 12, 6226 (2010).

${ }^{46}$ X.-P. Xing, X.-B. Wang, and L.-S. Wang, J. Phys. Chem. A 114, 4524 (2010).

${ }^{47}$ B. Baguenard, J. C. Pinaré, C. Bordas, and M. Broyer, Phys. Rev. A 63, 023204 (2001).

${ }^{48}$ B. Baguenard, J. C. Pinaré, F. Lépine, C. Bordas, and M. Broyer, Chem. Phys. Lett. 352, 147 (2002).

${ }^{49}$ K. Hansen, K. Hoffmann, and E. E. B. Campbell, J. Chem. Phys. 119, 2513 (2003).

${ }^{50}$ M. Kjellberg, O. Johansson, F. Jonsson, A. V. Bulgakov, C. Bordas, E. E. B. Campbell, and K. Hansen, Phys. Rev. A 81, 023202 (2010).

${ }^{51}$ N. Basov, E. Markin, A. Oraveski, A. Pankrato, and A. Skachkov, JETP Lett. 14, 165 (1971).

${ }^{52}$ D. P. Little, J. P. Speir, M. W. Senko, P. B. O'Connor, and F. W. McLafferty, Anal. Chem. 66, 2809 (1994).

${ }^{53}$ S. Ard, N. Mirsaleh-Kohan, J. D. Steill, J. Oomens, S. B. Nielsen, and R. N. Compton, J. Chem. Phys. 132, 094301 (2010).

${ }^{54}$ H. J. Cooper, K. Hakansson, and A. G. Marshall, Mass Spectrom. Rev. 24, 201 (2005) 